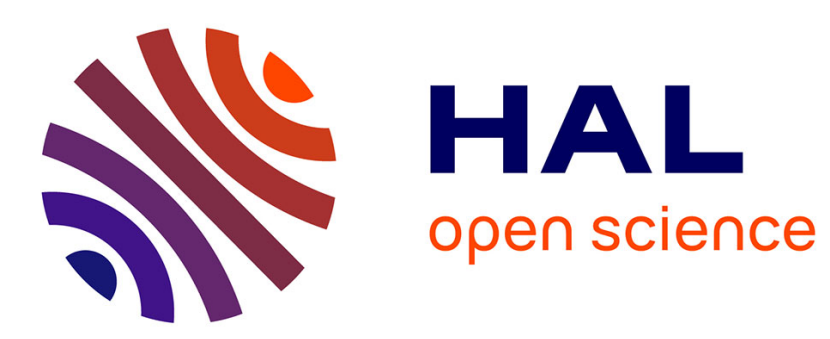

\title{
Alimentation hivernale des brebis laitières : intérêt de la mise en lots
}

Francois Bocquier, P. Guillouet, F. Barillet

\section{To cite this version:}

Francois Bocquier, P. Guillouet, F. Barillet. Alimentation hivernale des brebis laitières : intérêt de la mise en lots. Productions Animales, 1995, 8 (1), pp.19-28. hal-00896100

\section{HAL Id: hal-00896100 \\ https://hal.science/hal-00896100}

Submitted on 1 Jan 1995

HAL is a multi-disciplinary open access archive for the deposit and dissemination of scientific research documents, whether they are published or not. The documents may come from teaching and research institutions in France or abroad, or from public or private research centers.
L'archive ouverte pluridisciplinaire HAL, est destinée au dépôt et à la diffusion de documents scientifiques de niveau recherche, publiés ou non, émanant des établissements d'enseignement et de recherche français ou étrangers, des laboratoires publics ou privés. 
INRA Prod. Anim., $1995,8(1), 19-28$
F. BOCQUIER, P. GUILLOUET*, F. BARILLET**

INRA Sous-Nutrition des Ruminants Theix, 63122 St Genès Champanelle

* INRA-SAGA Domaine de La Fage 12250 Roquefort

* INRA-SAGA Auzeville BP 2731326 Castanet-Tolosan Cedex

\section{Alimentation hivernale des brebis laitières : intérêt de la mise en lots}

\begin{abstract}
Pendant la phase hivernale d'élevage des brebis laitières, les apports alimentaires sont souvent calculés en fonction des besoins des brebis les plus productives. Le gaspillage de concentré qui en résulte est de moins en moins supportable financièrement par l'éleveur. Un moyen de mieux ajuster les apports aux besoins réels des animaux consiste à les regrouper en lots en fonction de leurs productions attendues.
\end{abstract}

Les recommandations alimentaires sont principalement conçues pour être appliquées à un animal représentatif plutôt qu'à un ensemble d'individus. Or, la tendance actuelle en élevage des ruminants et surtout en élevage ovin, viande ou lait, est à l'accroissement

\section{Résumé}

Les techniques simplifiées d'alimentation collective mettent rarement en péril la santé des brebis ou leur productivité apparente, car de nombreuses régulations physiologiques leurs permettent de s'adapter aux excès ou aux déficits alimentaires. Mais elles conduisent à un gaspillage d'aliments concentrés ou à une baisse des production selon le niveau initial de performance de chaque brebis. La mise en lots des animaux en fonction de leurs besoins alimentaires est un moyen de mieux ajuster les apports alimentaires aux performances de production attendues. Les principaux facteurs de variation des besoins sont décrits et leur intérêt comme critère pour la constitution de lots homogènes est discuté. Deux essais de mise en lots ont été réalisés. Le premier comparait deux troupeaux de performances moyennes identiques, l'un dans lequel les brebis étaient conduites ensemble (Mélangées, $\mathrm{n}=96$ ), l'autre dans lequel les brebis étaient réparties en deux lots selon leur production laitière (Séparées-Bas, $n=48$ et Séparées-Haut, $n=48$ ). Au sein de chaque groupe les apports en concentrés ont été ajustés pour satisfaire les besoins de $85 \%$ des brebis, les fourrages étant distribués à volonté. Après 100 jours d'essai les consommations totales de fourrages et de concentrés ont été identiques ainsi que les productions laitières moyennes (Mélangées : 171 vs Séparées : $175 \mathrm{l} / 100$ j). Pour économiser des concentrés, il aurait fallu diminuer plus fortement les apports aux brebis du lot Bas. C'est ce que nous avons fait dans un deuxième essai où les apports de concentrés ont été soit identiques pour toutes les brebis (conduite classique, $n=67$ ), soit ajustés selon la production laitière, grâce à un distributeur automatique de concentré. Sur la période expérimentale, les productions laitières moyennes ont été identiques (Classique : $284,6 \mathrm{l} / 180 \mathrm{j}$ vs Ajusté : $282,2 \mathrm{~V} / 180 \mathrm{j}$ ), avec une distribution plus faible de concentré de $51 \mathrm{~kg}$ par brebis lorsque les apports sont ajustés. Les perspective d'utilisation de telles techniques d'alimentation en lot dépendront des développements de l'identification électronique et de l'automatisation du contrôle de performances. de la taille des troupeaux et par conséquent à l'hétérogénéité des performances des animaux conduits par éleveur. Ainsi, en élevage de brebis laitières, l'effectif atteint fréquemment 400 à 500 brebis (Barillet et Bocquier 1993). Ces effectifs importants devraient conduire l'éleveur à constituer des lots d'animaux en fonction de leurs exigences nutritionnelles afin d'ajuster au mieux les apports alimentaires. Mais l'affectation des animaux dans un lot nécessite de disposer de critères permettant de réduire la variabilité des besoins des différentes brebis du lot. Ces critères doivent englober les facteurs de variation des performances les plus importants, tant à l'échelle de l'individu au cours du temps qu'à l'échelle du troupeau à un moment donné. Ces facteurs de variation, abondamment étudiés chez la vache laitière, n'ont pas toujours la même importance chez la brebis laitière du fait des spécificités de la production de lait de brebis. Ainsi, par exemple, la collecte du lait, exclusivement destiné à la production de fromage, est limitée à la période d'ouverture des fromageries. La reproduction doit donc être conduite de telle sorte que le maximum de brebis soient mises à la traite à l'ouverture de la laiterie. De plus, des caractéristiques de l'espèce ovine, comme la prolificité, génèrent des écarts de besoins importants entre individus d'un même troupeau. La première partie de l'article présente donc la variabilité des besoins et de leur couverture dans le cas spécifique de la brebis laitière et de sa conduite d'élevage. La deuxième partie, après avoir présenté les conséquences de la dispersion 
des performances, propose les critères de mise en lots utilisables et rapporte les résultats de deux essais d'application.

\section{1 / Variations des besoins alimentaires et de leur satisfaction}

Chaque paragraphe présente les effets, à l'échelle de l'individu, des principaux facteurs de variation des besoins et les conséquences qui en découlent en situation d'élevage.

\section{1 / L'âge et le stade physiologique}

Au cours du cycle de production d'une brebis laitière les besoins évoluent quantitativement de manière différente pour l'énergie (UFL) et les protéines (PDI) : le rapport PDI/UFL de la ration doit donc être modulé au cours du cycle (figure 1a) (INRA 1988). De plus, comme les rations sont souvent constituées d'un maximum de fourrage, l'évolution de la capacité d'ingestion de la brebis au cours du cycle a souvent un effet déterminant. La densité énergétique et protéique de la ration devra évoluer pour satisfaire les besoins de brebis nourries ad libitum (figure 1b), on parlera alors d'exigences nutritionnelles plutôt que de besoins.
L'âge de la brebis est également un facteur de variation des exigences nutritionnelles. En effet, à même performances zootechniques, les primipares ont une capacité d'ingestion plus faible que celle des brebis plus âgées (Bocquier et al 1987a), alors que leur croissance n'est pas achevée. L'existence simultanée des besoins d'entretien, de croissance et de production de lait et d'une plus faible capacité d'ingestion accroît encore la dispersion des exigences nutritionnelles à l'échelle du troupeau dans lequel les primipares représentent fréquemment $30 \%$ de l'effectif.

\section{Gestation - Mise bas - Début de lactation}

Chez les brebis, l'évolution des exigences nutritionnelles avec l'avancement de la gestation varie fortement selon la taille de la portée. Plus celle-ci augmente et plus la capacité d'ingestion diminue rapidement (Thériez et al 1987) alors que l'augmentation des besoins s'accélère. Cette situation est très fréquente : dans un troupeau ayant une prolificité de $160 \%$, les proportions de brebis ayant des portées simples ou doubles sont voisines (figure 2). La mise en lot des brebis suivant la taille de la portée déterminée par échographie, permet de limiter la distribution d'aliment concentré aux brebis simples et de réduire les risques de toxémie de gestation chez les brebis portant deux ou trois agneaux (Brelurut et Thériez, comm. pers.).

Figure 1. Evolution, au cours du cycle de production d'une brebis laitière, des recommandations énergétiques et protéiques (a) et de la densité nutritive de rations permettant la couverture des recommandations alimentaires (d'après Bocquier et Caja 1993).
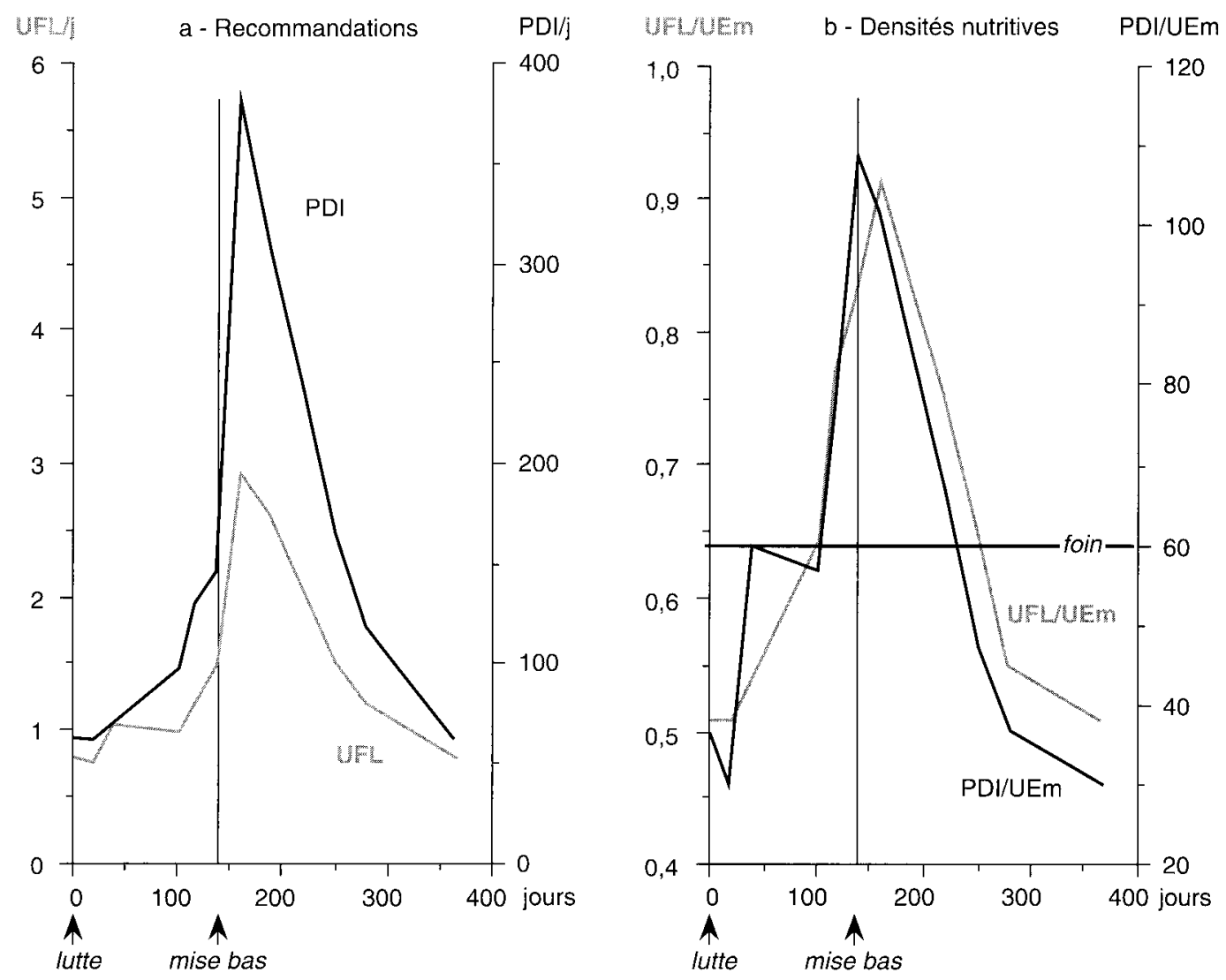
Figure 2. Evolution de la proportion de brebis ayant des portées de tailles différentes suivant la prolificité du troupeau (Bocquier et Thériez, non publié).

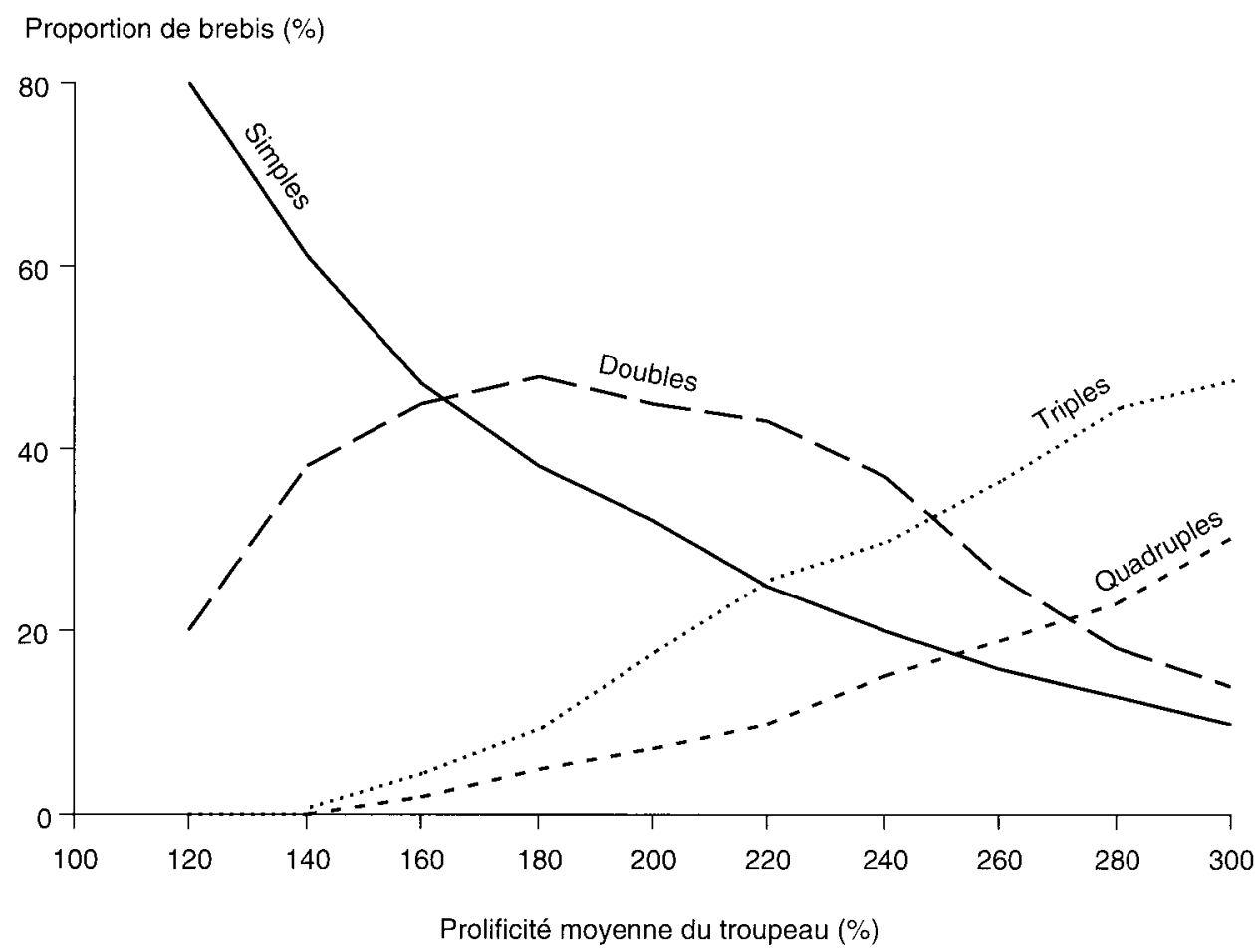

Au sein d'un troupeau, et bien que les pratiques modernes de maîtrise de la reproduction (synchronisation des œestrus, Courot et Volland-Nail 1991) soient fréquemment utilisées, la diversité des stades physiologiques des brebis présentes est le premier facteur d'hétérogénéité des besoins. En effet, même en regroupant les mises à la reproduction, les agnelages s'étalent souvent sur plus d'un mois, et comme la phase d'allaitement des agneaux dure 4 semaines, le troupeau peut être constitué, en période hivernale pour $1 / 3$ de brebis en fin de gestation, $1 / 3$ de brebis à l'allaitement et $1 / 3$ de brebis à la traite. Cette situation, fréquente dans les élevages des Pyrénées Atlantiques, est moins marquée dans le bassin de Roquefort.

Les techniques d'élevage mises en œuvre autour de l'agnelage (isolement des brebis dans des cases d'agnelage) facilitent en général le passage d'un régime alimentaire de fin de gestation à un régime de début de lactation. D'autres solutions ont été proposées, en élevage bovin allaitant notamment, où le changement de ration est déclenché lorsque la proportion de vaches ayant vêlé est voisine d'un tiers (Petit et al 1988). Cette règle simple est en général suffisante parce que les besoins des vaches allaitantes évoluent peu de part et d'autre du vêlage. Ce n'est pas le cas en élevage ovin où l'augmentation des besoins après la mise bas est très importante. Pour les brebis allaitantes, une mise en lots grossière peut être suffisante puisque le rationnement va durer peu de temps ( 6 à 8 semaines); elle doit être plus précise pour les brebis laitières puisque la période hivernale de rationnement peut durer jusqu'à 20 semaines.

\section{Période de traite}

Chez la brebis laitière, après le sevrage des agneaux à l'âge de 4 semaines, la production laitière brute diminue de manière quasi linéaire avec l'avancement de la lactation. On observe simultanément un enrichissement du lait en matières grasses et protéiques, dont les teneurs évoluent respectivement, en moyenne en race Lacaune, de 55 à $95 \mathrm{~g} / \mathrm{l}$ et de 45 à $65 \mathrm{~g} / \mathrm{l}$ du $2^{\circ}$ au $7^{\circ}$ mois de lactation (Barillet 1985, Bocquier et al 1987b). La diversité des stades de lactation a donc un effet très marqué sur la production : à un mois d'intervalle, des brebis ayant eu la même production laitière initiale (par exemple 2,0 1) peuvent présenter un écart de production laitière brute de près de 0,51 , soit $25 \%$. Compte tenu de l'enrichissement du lait, sur la base de l'énergie exportée (Bocquier et al 1993a) l'écart est cependant moindre $(18 \%)$. Le classement des brebis sur la production laitière brute est donc d'autant plus incertain, en termes de besoins énergétiques, que les stades de lactation sont dispersés et que les productions laitières initiales sont différentes.

Une étude conduite sur 150 élevages du Rayon de Roquefort a montré que la plus ou moins grande maittrise de la reproduction, et donc de l'étalement des stades de lactation, provoque de larges variations dans les volumes et dans la composition du lait livré à l'échelle de la campagne (Lagriffoul 1989). Ainsi, jusqu'à $45 \%$ des écarts entre producteurs sont expliqués par 2 paramètres : la proportion des brebis présentes en traite exclusive dès le premier jour de livraison (en moyenne $38 \%: \operatorname{mini}=14 \%$ et $\operatorname{maxi}=62 \%$ ),

\section{Au sein d'un troupeau, la diversité des stades physiologiques est le premier facteur d'hétérogénéité des besoins alimentaires.}


et l'étalement des entrées à la traite : du début de la traite jusqu'au moment où presque toutes les brebis sont traites il s'écoule de 30 à 120 jours (en moyenne 66 j).

\section{2 / Le niveau de performance}

Parmi les facteurs de variation liés à l'animal, les caractéristiques génétiques (race ou niveau génétique) influencent fortement le format de la brebis, ses performances de reproduction (prolificité) et de production laitière.

Les races de brebis laitières exploitées en France présentent des prolificités moyennes différentes entre elles (sans que l'on puisse estimer la part imputable à la race et aux systèmes d'élevage, largement confondus entre eux). Par exemple, en œstrus naturel, la prolificité moyenne des élevages en contrôle laitier officiel (source INRA-CTIG) avoisine $145 \%$ en race Lacaune, $120 \%$ pour les Manech et $110 \%$ pour les brebis Corses. Mais, comme il est rare qu'un élevage regroupe des brebis de plusieurs races, la nécessité de prendre en compte le type génétique dans le rationnement ne se rencontre généralement pas.

Intra-race, l'amélioration génétique des performances laitières au fil des années est une source non négligeable d'accroissement de l'hétérogénéité des productions intra-élevage. Ainsi en race Lacaune, le gain phénotypique moyen annuel est proche de $4 \%$ (décennie 80), les deux tiers de cette progression étant imputables au progrès génétique et un tiers à l'amélioration des conduites d'élevage (Barillet et al 1992 et 1993). L'accroissement continu du potentiel laitier moyen d'une population se traduit par une augmentation de la variabilité des performances laitières, étant donné que le coefficient de variation phénotypique reste stable et voisin de $28 \%$. Ainsi, pour les primipares de race Lacaune, la production laitière moyenne au premier contrôle laitier est passée de 1,35 l en 1980 à $1,78 \mathrm{l}$ en 1988 , ce qui a induit une augmentation de l'écart type de la production laitière au premier contrôle laitier de 0,38 à $0,50 \mathrm{l}$ (Barillet 1989). L'accroissement du potentiel génétique laitier génère donc à la fois l'accroissement des besoins et l'augmentation de la variabilité individuelle des performances. Il s'agit d'un phénomène général, constaté pour toutes les populations laitières en progression génétique. Cet effet va se trouver amplifié par les facteurs responsables de l'hétérogénéité des performances intra-élevage. Ainsi, par exemple, l'écart de production entre primipares d'un an et brebis adultes (3ème lactation et plus), voisin de $30 \%$, est passé de 20-30 l par lactation au début des années 80 à 40-60 l à la fin des années 80 .

En race Lacaune, les critères de sélection ont évolué récemment pour tenir compte de la composition du lait. De 1987 à 1992, la sélection a porté sur la quantité de "matière utile ", qui est une combinaison linéaire des quantités de matière grasse et de protéine, assimilable à un lait standard pour sa composition (Barillet et Boichard 1987). Depuis 1992 , outre le critère "quantité de matière utile ", l'accent a été mis sur le taux protéique (Barillet et al 1993). Le choix des critères de sélection a ainsi évolué pour tenter de stabiliser les taux tout en continuant d'accroître la quantité de lait produite. Néanmoins, à l'échelle du troupeau, le principal facteur de dispersion des besoins reste toujours la production laitière.

L'accroissement de la production laitière s'accompagne vraisemblablement de changements dans la partition des nutriments entre lait et réserves corporelles. Chez la vache laitière, on admet généralement que plus les animaux sont sélectionnés sur la production laitière et plus les nutriments sont orientés préférentiellement vers la mamelle. Les études en cours sur l'efficacité alimentaire de deux lignées divergentes de brebis Lacaune (Barillet et Bocquier 1993, Marie et al 1993) devraient permettre de préciser ces relations chez la brebis laitière.

\section{3 / Variabilité individuelle résiduelle}

L'étude des effets de l'alimentation sur les performances prend en compte tous les paramètres connus (race, stade physiologique, niveau de production, etc.) qui conditionnent les besoins d'un animal, permettant ainsi de définir des apports alimentaires recommandés. Néanmoins, même en tenant compte de tous ces critères, il subsiste des écarts entre performances prédites et performances observées. Ces écarts non expliqués sont regroupés sous le terme "variabilité résiduelle" dont l'amplitude est exprimée soit par l'écart-type résiduel (ETR), soit par le coefficient de variation résiduel (CVR), pour comparer les expérimentations entre elles (ETR/moyenne).

La variabilité résiduelle constatée lors d'expérimentations peut être élevée. Ainsi, bien qu'exprimés par $\mathrm{kg}$ de poids métabolique, les besoins d'entretien pour l'énergie et pour les protéines ont des coefficients de variation résiduels de l'ordre de $20 \%$ (Thériez et al 1987). Chez la brebis allaitante, la variabilité des quantités ingérées est encore plus importante (CVR : $47 \%$ ) même après avoir tenu compte du poids de la brebis, de son âge, de son état d'engraissement, de la croissance de sa portée et du niveau de complémentation (Bocquier et al 1987b). Bien que relativement élevés, ces coefficients de variation sont probablement sous-estimés car ils sont obtenus à partir de dispositif's expérimentaux dans lesquels les animaux sont souvent plus homogènes qu'en élevage. La conséquence pratique de cette variabilité résiduelle est qu'il est difficile de déterminer avec précision le besoin réel de chaque individu même lorsqu'on connaît ses caractéristiques zootechniques.

Pendant la lactation, le mauvais ajustement des apports alimentaires aux besoins individuels en situation d'alimentation collective aboutit rarement à des troubles métaboliques. En effet, les ruminants en lactation sont capables de s'adapter à une sur- ou une sousalimentation très importante (Bauman et 
Curie 1980, Chilliard 1988). Cette adaptation est efficace, comme le montrent, a posteriori, les relations très précises entre les termes du bilan énergétique (Energie ingérée, E lait, Ecorporelle, E entretien) (Moe et al 1971, INRA 1978, Saama et Mao 1993). Mais on ne peut prédire l'évolution des éléments du bilan énergétique que si les animaux sont alimentés au voisinage des recommandations, ce qui concerne peu d'individus en élevage de brebis laitières. Aussi, avec les mêmes caractéristiques zootechniques individuelles initiales et les mêmes apports alimentaires, l'évolution des performances et l'utilisation de l'énergie corporelle peuvent largement différer entre animaux. Cette évolution particulière à chaque animal fait que la variabilité interindividuelle des performances augmente avec le temps, et que sa prise en compte nécessite des contrôles zootechniques fréquents.

Les travaux entrepris sur la modélisation des réponses des vaches laitières à l'alimentation (Oldham et Emmans 1989, Black et al 1993) devraient permettre de mieux prédire l'évolution du bilan énergétique, mais ils n'ont pas encore été entrepris pour les brebis laitières.

\section{2 / La mise en lots : un moyen de réduire la variabilité}

\section{1 / Conséquences de la dispersion des performances}

Dans un élevage, on peut considérer, pour simplifier, que la répartition à un moment donné des productions laitières (qui conditionne principalement les besoins) suit une loi normale caractérisée par sa valeur moyenne (PLm) et son écart type (ECT) (figure 3). Deux troupeaux peuvent avoir la même production laitière moyenne, mais différer par la dispersion des performances. Intra-troupeau, les coefficients de variation des performances laitières varient dans une plage de 20 à $40 \%$. Si la ration couvre les besoins correspondant à la production laitière moyenne plus un écart-type (PL-objectif : PLo $=$ PLm + ECT), les besoins de $83 \%$ des brebis sont au moins couverts. Les situations nutritionnelles sont néanmoins très contrastées: les brebis les plus performantes sont insuffisamment nourries, mais elles sont peu nombreuses (moins de $17 \%$ ), inversement les brebis les moins performantes sont largement suralimentées, et elles sont beaucoup plus nombreuses (plus de $50 \%$ ).

La contribution relative des différentes classes de production laitière à la production totale du troupeau est très inégale et les classes de brebis qui contribuent le plus à la production totale sont situées à environ $10 \%$ au dessus de la classe moyenne (figure 3). En l'absence de mise en lots, le rationnement le plus simple consisterait à distribuer aux brebis une ration qui couvrirait les besoins de cette classe de brebis la plus importante vis-à-vis de la production laitière totale du troupeau.
Figure 3. Courbe de répartition des productions laitières des brebis dans un troupeau : incidence du choix d'un objectif de production laitière sur la satisfaction des besoins des différentes brebis.

Paricipation a la

$\%$ brebis production tature toble ou rouperis

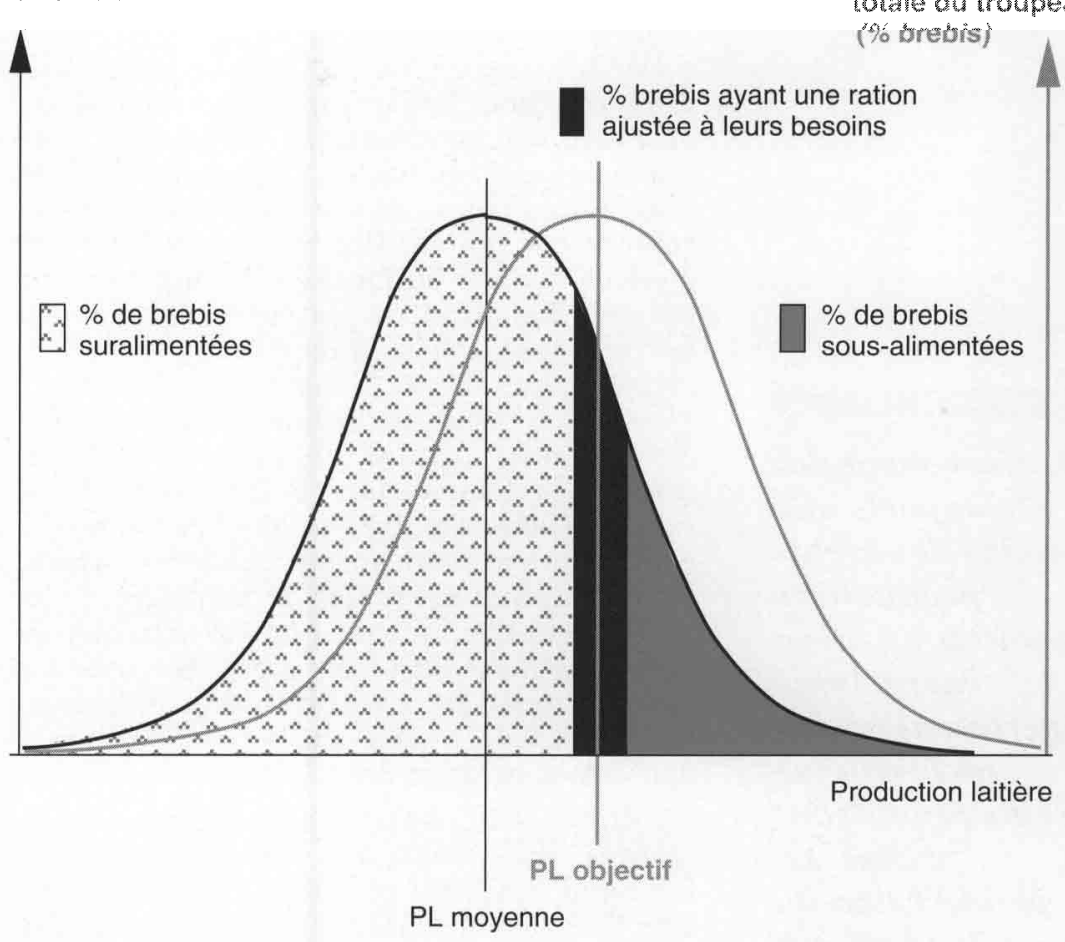

Pour un objectif de production donné et avec des apports de concentré identiques pour toutes les brebis, ce qui est fréquent lors des distributions en salle de traite, le bilan énergétique théorique diminue régulièrement lorsque la classe de production laitière augmente (figure 4). Si l'on pondère le bilan énergétique par les effectifs de brebis dans chaque classe de production, les zones d'excédents énergétiques importants apparaissent clairement (figure 4). Cette inadéquation des apports aux besoins énergétiques se traduira par un accroissement de l'état corporel chez les faibles productrices (proportion élevée) et par une mobilisation des réserves chez les brebis les plus performantes (proportion faible).

De plus, si la ration de base est encombrante, elle sera complémentée par des apports importants d'aliments concentrés, et le gaspillage de concentré sera encore plus important qu'avec une ration de bonne ingestibilité (figure 4).

Ce raisonnement concernant les apports d'énergie ne s'applique pas de la même façon à la nutrition protéique car, en cas de déficit, les possibilités d'utilisation des protéines corporelles sont faibles (Bocquier et al 1987a). Il faudrait donc accroître la PL-objectif lorsque la densité protéique de la ration de base est faible. Cette situation est très fréquente dans les élevages de brebis laitières (Vacaresse et Arranz 1993) où les apports azotés sous forme de concentré constituent une sécurité pour le maintien de la production de lait et de
La classe de brebis plus à la production laitière totale du troupeau est celle produisant $10 \%$ de plus que la production laitière moyenne. Si l'alimentation du troupeau entier est calculée en fonction des besoins de ces brebis, plus de la moitié du troupeau est suralimenté. qui contribue le 
matières protéiques, ce qui se traduit par un gaspillage de protéines alimentaires encore plus important que pour l'énergie.

\section{2 / Choix des critères et du nombre de lots}

Dans la réalité, les courbes de répartition des productions laitières intra-troupeau (à une date donnée) sont souvent bimodales, voire multimodales, selon les vagues successives d'agnelage (brebis adultes, primipares et retours), ce qui facilite la constitution d'au moins deux lots en fonction de la production laitière. Mais d'autres facteurs conditionnent les quantités ingérées de fourrages, notamment le poids vif des brebis et leur état corporel. Ainsi, à même production laitière, une brebis de petit format devrait être placée préférentiellement dans le lot recevant une ration de densité nutritive élevée par rapport à celle qui, ayant un format plus important, pourra ingérer davantage de fourrages. Cette notion sera d'autant plus importante que les brebis les moins bien alimentées recevront une ration permettant une PL-objectif nettement inférieure à la production laitière correspondant à la séparation des 2 lots.

Afin de réduire l'hétérogénéité des performances entre brebis d'un même troupeau on a simulé, avec des résultats du contrôle laitier, l'effet de la séparation des brebis en plusieurs lots selon leurs productions laitières (Vacaresse 1992). Le nombre de lots a une influence déterminante sur la réduction de l'hétérogénéité. Par rapport à un indice d'hétérogénéité initiale de 100 , la constitution de deux lots est très efficace $(-24 \%)$, en revanche le passage de deux lots à trois lots est moins efficace $(-6 \%)$ et celui de trois à

Figure 4. Evolution du taux de couverture des besoins dans chacune des classes de production laitière, selon l'encombrement de la ration de base.

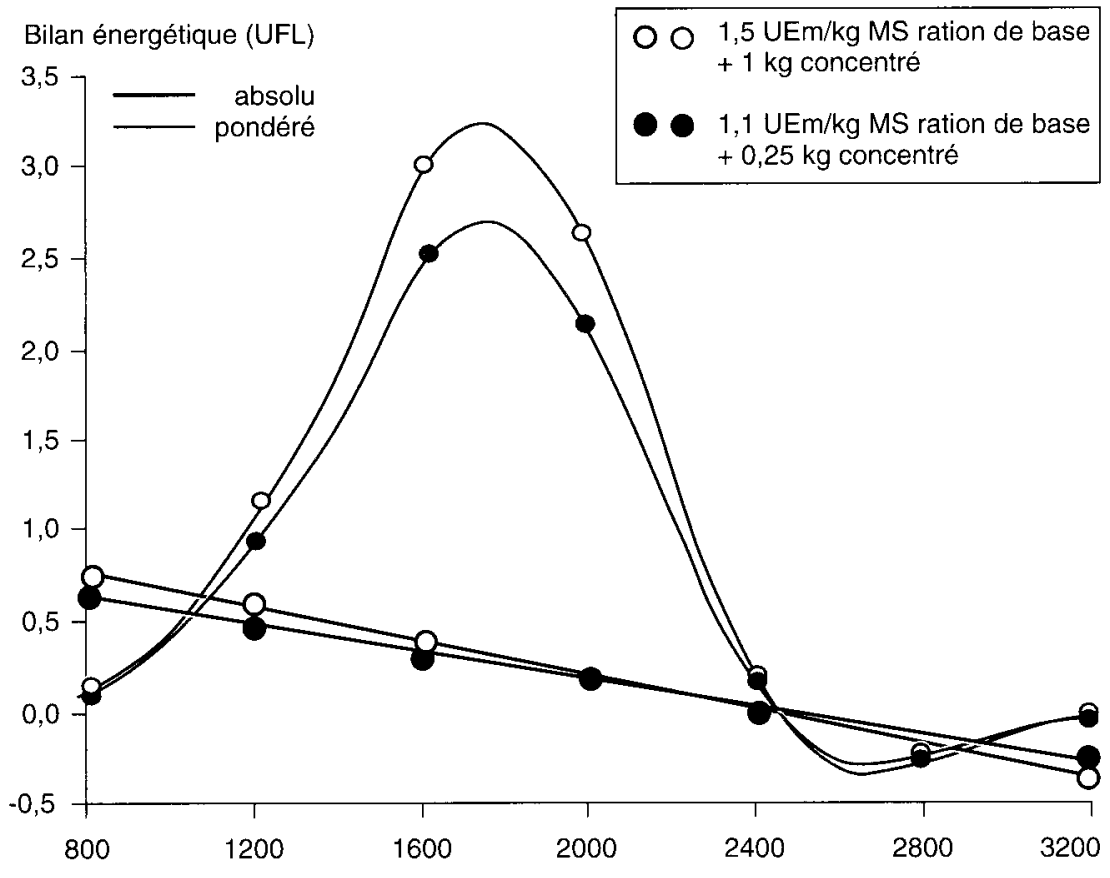

Classes de production laitière $(\mathrm{mg} / \mathrm{j})$ quatre lots l'est encore moins (-2\%). De plus, la proportion d'animaux affectés à chaque lot a également un effet sur la réduction de l'hétérogénéité initiale: le découpage en deux lots de taille égale permet une réduction maximale. Au-delà de deux lots, tous les systèmes équilibés (3 lots d'1/3 de l'effectif, 4 lots d'1/4) sont ceux qui permettent les réductions les plus importantes d'hétérogénéité.

D'autres simulations réalisées pour des vaches laitières (Clarck et al 1978, Pecksoc et al 1992) aboutissent à des conclusions analogues : il est d'autant plus intéressant de faire des lots d'alimentation que le niveau génétique des vaches est plus élevé ( 24 ou $271 / \mathrm{j}$ ) et/ou que les stades de lactation sont plus ou moins dispersés (CV des dates de vêlage $8 ; 15$ et $20 \%$; Pecksoc et al 1992). Il a été montré, comme pour les brebis laitières, que lorsqu'on utilise la même quantité totale d'aliments, la séparation d'un troupeau en deux lots permet d'espérer un gain de production laitière de $3 \%$, et qu'entre deux et trois lots le gain de production laitière est moindre $(+2 \%)$.

De plus, McGilliard et al (1983) ont comparé, sur 80 troupeaux de vaches laitières, différentes méthodes pour regrouper les vaches en lots d'alimentation. Il apparaît que les méthodes de classification automatique sur les critères de densité énergétique et protéiques nécessaires à chaque animal seraient nettement plus efficaces que les productions laitières individuelles brutes ou standardisées, ou que les index laitiers. Pour deux lots d'alimentation, en comparant les classifications simplifiées (PLB, PLS ou Index) aux résultats de classification automatique, les proportions de vaches mal classées seraient respectivement de 25,22 et $15 \%$. L'intérêt des différents critères de mise en lot cités cidessus a été ré-examiné et confirmé par Williams et Oltenacu (1992); ils seront évalués lors de prochains essais sur brebis laitières.

\section{3 / Séparation d'un troupeau en deux lots}

Au domaine INRA de la Fage (Guillouet $e t$ al 1992a), deux petits troupeaux de 96 brebis ont été constitués en fonction de la production laitière, du poids vif initial et de la note d'état corporel par la méthode des couples. Les brebis du premier troupeau ont été alimentées ensemble (conduite mélangée $\mathrm{n}=96$ ), celle du second en deux lots séparés (Séparé Haut $n=48$ et Séparé Bas, $n=48$ ). Les mesures ont porté sur les 100 premiers jours de traite exclusive après le sevrage des agneaux (29 au 129e jour de lactation). La tactique alimentaire appliquée dans tous les cas était de couvrir les besoins d'environ $85 \%$ des brebis de chaque lot. Les réajustements de rations ont été effectués simultanément dans les trois lots (toutes les 3 semaines) en modifiant les apports d'aliments concentrés. En moyenne, la ration de base était constituée (en \% MS) d'un mélange de foin de luzerne $(23 \%)$, d'ensilage de luzerne $(47 \%)$, de pulpe 
Tableau 1. Apports alimentaires et performances de production (en moyenne par brebis pour 100 jours) des brebis Lacaune selon leur mode de conduite.

\begin{tabular}{|l|c|c|c|c|c|c|}
\hline \multirow{2}{*}{ Conduite } & \multicolumn{3}{|c|}{ mélangée } & \multicolumn{3}{c|}{ séparée } \\
\cline { 2 - 7 } & Lot Bas & Lot Haut & Ensemble & Lot Bas & Lot Haut & Ensemble \\
\hline Effectif & 48 & 48 & 96 & 48 & 48 & 96 \\
\hline Aliments (g MS/b/j) & & & & & & \\
- ration de base & nd & nd & 3150 & 2830 & 3430 & 3130 \\
- concentré & nd & nd & 312 & 187 & 478 & 330 \\
\hline Lait produit (l/j) & 1,35 & 2,07 & 1,71 & 1,36 & 2,15 & 1,75 \\
Taux butyreux (g/l) & 65,1 & 62,7 & 63,9 & 63,3 & 62,6 & 63,0 \\
Taux protéique (g/l) & 51,2 & 49,9 & 50,9 & 51,8 & 49,6 & 50,7 \\
\hline Poids vif $(\mathrm{kg})$ & & & & & & 78,9 \\
- début $(\mathrm{j}+2)$ & 77,7 & 79,4 & 78,5 & 78,7 & 79,0 & 78,9 \\
- fin $(\mathrm{j}+77)$ & 81,5 & 80,9 & 81,2 & 83,3 & 83,2 & 83,2 \\
\hline
\end{tabular}

de betterave déshydratée $(15 \%)$ et de luzerne déshydratée (15\%). Les consommations globales de fourrage et d'aliments concentrés (tableau 1) ont été identiques pour les deux troupeaux. Compte tenu de la valeur nutritive de la ration de base, la distribution d'aliment concentré a été supprimée dans tous les lots à partir du $87^{\mathrm{e}}$ jour de lactation.

Globalement les productions laitières moyennes de ces deux troupeaux n'ont pas été significativement différentes (175 (conduite séparée) vs 171 (conduite mélangée) litres/brebis en 100 jours de traite). La conduite en lots séparés semble avoir provoqué une baisse du taux butyreux du lait en fin d'essai dans le lot Bas, sans affecter le taux protéique (tableau 1). Dans tous les cas, la couverture des besoins de $85 \%$ des brebis de chaque lot a entraîné un accroissement du poids vif et de l'état corporel moyens des brebis, accroissement significativement plus important dans le lot Séparé-Bas.

La constitution de deux lots séparés a, dans un premier temps, réduit l'hétérogénéité relative initiale des productions laitières (S-Haut $\mathrm{CV}=15,7 \%$ et $\mathrm{S}-\mathrm{Bas} 20,1 \%$ ) par rapport à celle observée dans le troupeau $(25 \%)$. Mais l'évolution des productions laitières avec l'avancement de la lactation a accru l'hétérogénéité dans chacun des lots, particulièrement pour les brebis faibles productrices (lot S-Bas). On assiste progressivement à un chevauchement partiel des distributions de productions laitières des lots S-Bas et S-Haut (figure 5).

Cet essai montre qu'on peut obtenir des performances zootechniques équivalentes en maîtrisant la conduite en lots, lorsque les tactiques d'alimentation sont identiques. En revanche, elle ne permet d'économiser des aliments concentrés que si les objectifs de production de chaque lot sont bien différenciés. Pour réaliser ces économies, il aurait fallu en outre ré-alloter les brebis à chaque contrôle laitier afin de maintenir une bonne homogénéité des performances dans chacun des lots. Ces éléments ont été intégrés dans l'expérimentation présentée ci-dessous.
Figure 5. Evolution de la distribution des productions journalières des lots bas et haut.
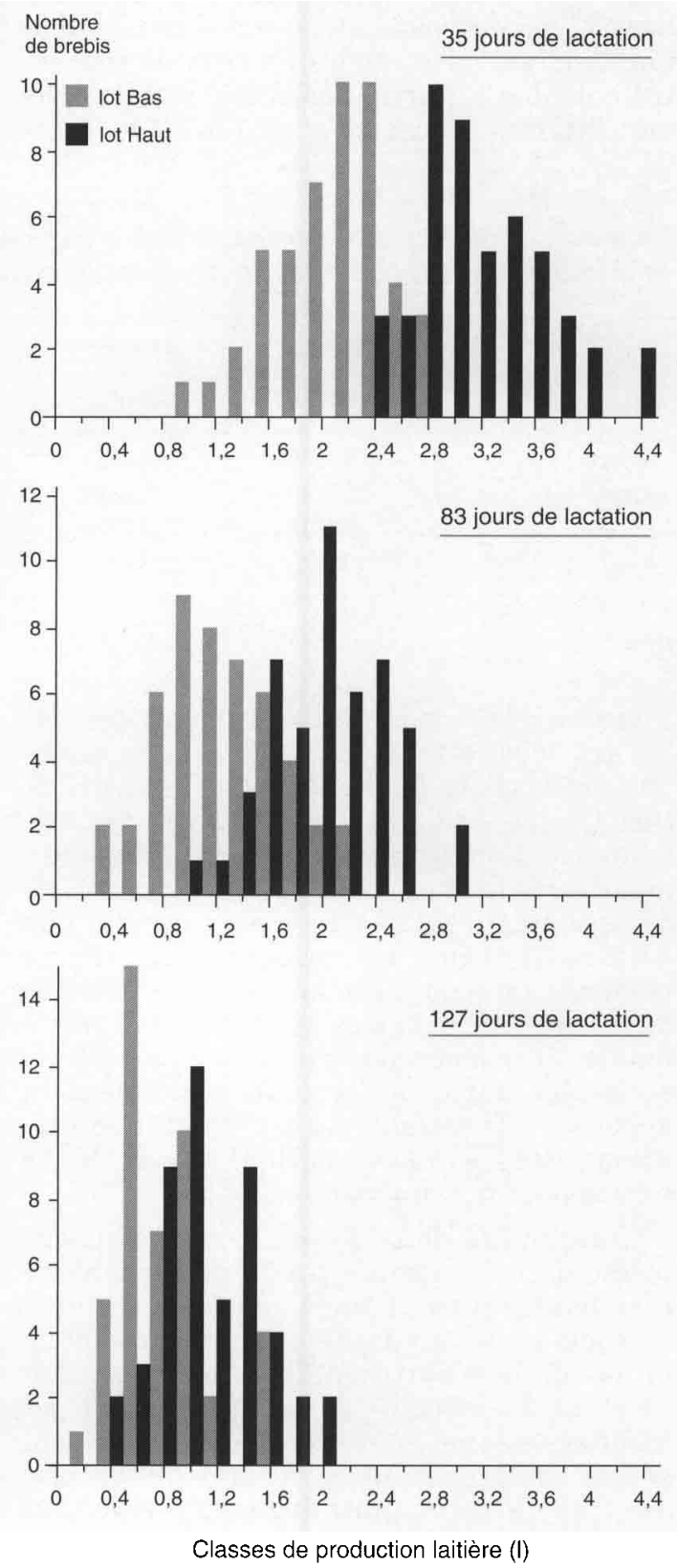

La mise en lots en fonction de la production laitière est inefficace lorsque les brebis sont maintenues dans le même lot tout au long de leur lactation. 


\section{4 / Utilisation d'un distributeur automatique de concentré}

L'utilisation de distributeurs automatiques de concentrés n'est pas fréquente dans les élevages de brebis laitières. Deux cas peuvent se rencontrer : la distribution contrôlée en salle de traite ou l'utilisation d'un dispositif en « self service " installé au milieu d'un lot de brebis (Guillouet et al 1992b).

La distribution contrôlée en salle de traite a fait l'objet d'une expérimentation dans un élevage privé (Vacaresse 1994), grâce à un système d'identification électronique. La distribution des aliments concentrés a été ajustée selon la production laitière. Ce mode de distribution a été comparé à une distribution classique où les consommations sont identiques pour toutes les brebis, les quantités distribuées dépendant des performances moyennes des meilleures brebis du lot.

Dans cet essai, les fourrages étaient distribués à volonté, sans mesure des consommations individuelles, et les apports de concentré calculés à partir des recommandations alimentaires (Bocquier et al 1987a) en fonc- tion de la production laitière et compatibles avec la capacité d'ingestion des animaux.

Des brebis (134) ont été appariées selon le stade de lactation et la production laitière. Elles ont été réparties dans les deux lots (Classique ou Ajusté) mais maintenues ensemble. Celles qui étaient en conduite Classique ont reçu, quelle que soit leur production laitière individuelle, les mêmes apports de concentrés. Ces apports ont diminué avec l'avancement de la lactation. Dans ce traitement les quantités distribuées sont passées progressivement de $0,78 \mathrm{~kg}$ MS/j au début de l'essai à 0,58 six mois plus tard. Les 67 brebis qui étaient dans le traitement Ajusté étaient réparties en 4 sous-groupes correspondant à 4 niveaux de distribution de concentré : les meilleures laitières recevaient pratiquement les mêmes apports que le lot en conduite Classique, alors que les 3 autres lots recevaient des apports plus faibles (une diminution moyenne de $0,2 \mathrm{~kg}$ par niveau). Au cours de l'essai, les effectifs de brebis dans ces différents sous-groupes ont évolué en fonction de leurs performances laitières mesurées lors de contrôles laitiers mensuels (tableau 2).

Tableau 2. Evolution des quantités brutes d'aliment concentré distribuées (g/j par brebis) selon le mode de distribution, classique ou ajusté (les effectifs de brebis sont notés entre parenthèses).

\begin{tabular}{|lllllll|}
\hline Durée $(\mathrm{j})$ & 0 à 30 & 31 à 60 & 61 à 90 & 91 à 101 & 102 à 120 & 121 à 152 \\
\hline Classique & $780(67)$ & $780(64)$ & $715(58)$ & $650(57)$ & $650(57)$ & $650(57)$ \\
\hline Ajusté 1 & $780(30)$ & $780(12)$ & $715(9)$ & $715(2)$ & $715(2)$ & $715(1)$ \\
Ajusté 2 & $585(18)$ & $585(21)$ & $520(8)$ & $520(8)$ & $520(8)$ & $520(3)$ \\
Ajusté 3 & $390(15)$ & $390(22)$ & $325(22)$ & $455(10)$ & $390(10)$ & $325(20)$ \\
Ajusté 4 & $195(4)$ & $195(9)$ & $130(19)$ & $255(37)$ & $195(37)$ & $130(33)$ \\
\hline
\end{tabular}

Les productions laitières moyennes des brebis des deux lots ont été identiques en distribution Classique $(284,6 \mathrm{l} / 180 \mathrm{j})$ et en distribution ajustée $(282,21 / 180 \mathrm{j})$. Cet ajustement de la distribution des aliments concentrés a permis de réaliser une économie moyenne de $51 \mathrm{~kg}$ de concentré par brebis pendant la période de traite. Une extrapolation de ces résultats, en tenant compte des différences de durée de traite et des productions individuelles, a permis d'estimer que l'économie réalisable par ce système de rationnement appliqué à l'ensemble du troupeau (398 brebis adultes +165 agnelles) serait de 18 tonnes pour la campagne.

L'originalité de ce mode de rationnement réside dans le nombre limité de niveaux de complémentation (4 seulement) qui évoluent au cours de la campagne pour tenir compte à la fois de la répartition des productions laitières et des effectifs de brebis. Même si les résultats obtenus sont spectaculaires, on peut penser intégrer d'autres critères que la production laitière pour limiter encore les apports de concentrés.

\section{Conclusions}

Pour faire face à l'hétérogénéité des performances des brebis laitières, la solution retenue conduit souvent à suralimenter les individus les moins performants. Mais le gaspillage d'aliments qui en résulte apparaît de moins en moins tolérable du point de vue économique. La mise en lots des brebis sur le critère de la production laitière brute est une première étape nécessaire pour mieux ajuster les apports alimentaires aux besoins des animaux. Actuellement, le logiciel INRAtion (Agabriel et al 1988-1993) permet d'intégrer les résultats du dernier contrôle laitier, de visualiser l'hétérogénéité des performances, de concevoir des lots homogènes sur la production laitière brute et de comparer les différents rationnements possibles. Pour prendre en compte des critères supplémentaires, il faudra ultérieurement envisager d'utiliser des méthodes de classifications automatiques. Les nouveaux systèmes d'identification électroniques testés chez les ovins laitiers, en particulier à La Fage (Astruc et al 
1992, Guillouet et al 1992b, Marie et al 1994), permettent d'envisager des distribution sélectives d'aliments concentrés (salle de traite ou via des distributeurs automatiques d'aliments concentrés en bergerie), voire même de la totalité de la ration (contrôle électroniques d'accès à des lots après la traite) comme cela est déjà envisagé pour les vaches laitières (Geers 1994). L'apport de l'électronique apparaît essentiel pour l'élevage des brebis laitières compte tenu des effectifs de brebis à la traite. Ces dispositifs et les modes de conduites des brebis qui peuvent en découler permettront probablement des économies importantes d'aliments et, en tout cas, un meilleur état nutritionnel des animaux par un ajustement des apports aux besoins. Il faudra toutefois comparer le coût des procédures de mise en lots (contrôles zootechniques) avec l'économie d'aliment concentré qui peut être réalisée.

\section{Remerciements}

Nous tenons à remercier le personnel de La Fage, en particulier Marie-Rose Aurel, pour le suivi efficace des essais, ainsi que les membres du Groupe technique Alimentation des brebis laitières du CNBL qui ont participé à la réflexion sur le sujet de cet article.

L'expérience réalisée à La Fage a été financée dans le cadre du programme CEE 8001 - CT91 - 0113.

\section{Références bibliographiques}

Agabriel J., Champciaux P., Espinasse C., Coulon J.B., Micol D., Bocquier F., Faverdin P., 1988-1993. INRAtion : Logiciel de référence pour le calcul et l'analyse des rations destinées aux bovins, ovins et caprins. CNERTA diffusion, 26 Bd Dr Petitjean, 21000 Dijon.

Astruc J.M., Arhainx J., Barillet F., Lagriffoul G., Guillouet P., Oberti J., Ricard E., 1992. Informatisation et automatisation du contrôle laitier ovin en France. 28th biennial Session of ICAR, Neustift im Stubaital, Tyrol, Austria, 7-12 June 1992, 95-102.

Barillet F., 1985. Amélioration génétique de la composition du lait des brebis : l'exemple de la race Lacaune. Thèse de Docteur-Ingénieur, INA ParisGrignon, 144 p. + annexes, Paris.

Barillet F., 1989. Expression de la production laitière à la traite des brebis Lacaune en système allaitement $\mathrm{x}$ traite mécanique. 4 Symposium international sur la traite des petits ruminants, Tel-Aviv, Israel, 13-19 septembre 1989, 463-495.

Barillet F., Bocquier F., 1993. Le contexte de production des ovins laitiers en France : principaux objectifs de recherche-développement et condition de leur mise en œuvre. INRA Prod. Anim., 6, 17-24.

Barillet F., Boichard D., 1987. Studies on dairy production of milked ewes. I. Estimates of genetic parameters for total milk composition and yield. Gen. Sel. Evol., 19, 459-474.

Barillet F., Boichard D., Barbat A., Astruc J.M., Bonaïti B., 1992. Use of an animal model for genetic evaluation of the Lacaune dairy sheep. Livest. Prod. Sci., 31, 287-299.

Barillet F., Sanna S., Boichard D., Astruc J.M. Carta M., Casu S., 1993. Genetic evaluation of the Lacaune, Manech and Sarda dairy sheep with animal model. Proc. of 5th Symposium Machine Milking of Small Ruminant. Budapest 15-19 Mai 1993. Hung. J. Anim. Prod., suppl 1, 289-310.

Bauman E., Currie W.B., 1980. Partitionning of nutrients during pregnacy and lactation; a review of mechanisms involving homeostasis and homeorhesis. J. Dairy Sci., 63, 1514-1529.

Black J.L., Davies G.T., Fleming J.F., 1993. Role of computer simulation in the application of knowledge to animal industries. Austr. J. Agric. Res., 44, 541555 .
Bocquier F., Caja G., 1993. Recent advances in dairy sheep nutrition. Proc. of 5th Symposium Machine Milking of Small Ruminant. Budapest 15-19 Mai 1993. Hung. J. Anim. Prod., suppl 1, 580-607.

Bocquier F., Thériez M., Brelurut A., 1987a. The voluntary hay intake of ewes during the first weeks of lactation. Anim. Prod., 44, 387-394.

Bocquier F., Thériez M., Brelurut A., 1987b. Recommandations alimentaires pour les brebis en lactation. Bull. Tech. CRZV-Theix, INRA, 70, 199-211.

Bocquier F., Barillet F., Guillouet P., Jacquin M., 1993a. Prévision de l'énergie du lait de brebis à partir de différents résultats d'analyses : proposition de lait standard pour les brebis laitières. Ann. Zootech., 42, 57-66.

Bocquier F., Guillouet P., Barillet F., Ligios S., Molle G., Sanna A., Casu S., Caja G., Such X., Gasa J., Ferret A., Oregui L., Urarte E., Agabriel J., Champciaux P., Espinasse C., 1993b. A computer program for diet formulation in dairy sheep : Evaluation of food intake predictions. In : Proc 5th International Symposium on Machine Milking of Small Ruminants. Budapest May 15-19 1993. Hung. J. Anim. Prod., 608-621.

Chilliard, 1988. Rôles et mécanismes d'action de l'hormone de croissance chez le ruminant en lactation. Reprod. Nutr. Develop., 28, 39-59.

Clarck P.W., Ricketts R.E., Beleya R.L., Krause G.F., 1978. Feeding and managing dairy cows in three versus one production group. J. Dairy Sci., 63, 12991308 .

Coppock C.E., 1976. Management of dairy cows in group housing. J. Dairy Sci., 60, 1327-1336.

Courot M., Volland-Nail P., 1991. Conduite de la reproduction des mammifêres domestiques : présent et futur. INRA Prod. Anim., 4, 21-29.

Geers R., 1994. Electronic monitoring of farm animals : a review of research and development requirements and expected benefits. Computers and Electronics in Agriculture, 10, 1-9.

Guillouet P., Bocquier F., Fraysse J., Jacquin M., Lagriffoul G., Barillet F., 1992a. Intérêt de l'alimentation par lots dans les troupeaux de brebis laitières Lacaune en bergerie. CR annuel contrat Camar CEE, DGVI, n' CT91-0113. 
Guillouet P., Ricard E., Arhainx J., Lagriffoul G., Barillet F., 1992b. Mise au point et utilisation d'un distributeur automatique d'aliment concentré par les brebis laitières. $\mathrm{CR}$ annuel contrat Camar CEE, DGVI, n" CT91-0113.

INRA, 1988. Alimentation des Bovins, Ovins et Caprins. Ed. R. Jarrige. INRA Publications 147 rue de l'Université Paris.

Lagriffoul G., 1989. Influence des conduites d'élevage sur la composition des laits de troupeaux de brebis Lacaune. INRA. INA-PG Mémoire de fin d'étude, $72 \mathrm{p}$.

Marie C., Guillouet P., Bocquier F., Jacquin M., Barillet F., 1993. Efficacité alimentaire pour la production laitière en race ovine Lacaune selon le niveau génétique laitier et la stratégie alimentaire. CR annuel de contrat CAMAR, CEE DG VI, n" CT91-0113.

Marie C., Caja G., Barillet F., Ribo O., Nehring R., Ricard E., 1994. Electronic identification in sheep : initial results and considerations for application and testing of transponders. Proc. 29th session of ICAR, Ottawa, Canada, 31 july-5 august 1994 (sous presse)

McGilliard M.L., Swisher J.M., James R.E., 1983. Grouping lactating cows by nutritionnal requirements for feeding. J. Dairy. Sci., 66, 1084-1093.

Oldham J.D., Emmans G.C., 1989. Prediction of responses to required nutrients in dairy cows. J. Dairy Sci., $72,3212-3229$.

Pecsok S.R., McGuilliard M.L., James R.E., Johnson T.G., Holter J.B., 1992. Estimating production bene- fits through simulation of group and individual feeding of dairy cows. J. Dairy Sci., 75, 1604-1615.

Petit et $a l, 1988$. Tables de rationnement des ruminants R. Jarrige ED. INRA publications.

Saama P.M., Mao I.L., 1993. Sources of variation in partitionning of intake energy for lactating Hostein cows. J. Dairy Sci., 76, 1334-1341.

Smith N.E., Ufford G.R., Coppock C.E., 1978. One versus two group system for lactating cows fed complete rations. J. Dairy Sci., 61, 1138-1145.

Thériez M., Bocquier F., Brelurut A., 1987. Recommandations alimentaires pour les brebis à l'entretien et en gestation. Bull. Tech. C.R.Z.V., Theix, INRA, 70, 185-197.

Mœ P.W., Tyrrell H.F., Flatt W.P., 1971. Energetics of body tissue mobilization. J. Dairy Sci., 51, 548553.

Vacaresse C., 1992. Constitution de lots pour l'alimentation des brebis laitières. SEB, 13, 4-5.

Vacaresse C., 1994. Essai de conduite en lots virtuels pour l'alimentation des brebis laitières. Pâtre (sous presse).

Vacaresse C., Arranz J.M., 1993. Enquêtes sur les tactiques alimentaires appliquées aux brebis laitières de l'Aveyron et des Pyrénées Atlantiques. Pâtre, 406, 29-32.

Williams C.B., Oltenacu P.A., 1992. Evaluation of criteria used to group lactating cows using a dairy production model. J. Dairy Sci., 75, 155-160.

\begin{abstract}
Grouping dairy ewes by nutritional requirements for winter feeding.

In large flocks of dairy ewes, classical concepts of animal feeding can no longer be applied because of the increasing variability of performances. Simplified feeding practices by means of generalised group-feeding rarely impair animal health and apparent productivity because many physiological regulations permit each animal to adapt to imperfect nutrient supply (either excess or deficit). However, in these conditions, predictions of ewe's performances are almost impossible since animal responses to such a variation in nutrient balance is mostly unknown. In this paper, main production factors accounting for the variability of requirements are described and their usefulness is discussed with respect to the efficiency of allocation criteria into smaller sized group-feeding.
\end{abstract}

Abstract

In a trial, two identical small flocks of 96 ewes were either conducted together (Mixed) or separated in two groups according to milk yield (Separated-High $\mathrm{n}=48$ and Separated-Low $n=48$ ). Within each group the feeding objective was to supply concentrates in order to satisfy $85 \%$ of the ewes, with forages available ad libitum. After 100 days, for each of the two treat- ments (Mixed vs Separated), mean consumptions were very close and mean milk yield were non significantly different (resp. 171 vs $175 \mathrm{l} / 100 \mathrm{~d}$ ). Concentrate distribution could have been decreased in Separated-Low group to earn concentrate. That's what was done in an on farm trial without control of forage intake. Comparison was made between a group $(n=67$ ewes) receiving classical amounts of concentrate (Classical) while the other group was divided in four sub-groups receiving adjusted amounts of concentrates according to their milk yield by means of an electronic feeder. On the whole experimental period, mean milk yield were the same (Classical 284.6 1/180 d vs Adjusted $282.2 \mathrm{l} / 180 \mathrm{~d}$ ), with a difference of $51 \mathrm{~kg}$ concentrate less per ewe for adjusted treatments. The future of such techniques, in order to increase nutrient efficiency and performance of dairy ewes, will depend on the development of electronic recognition devices (individual feeding, electronic gates) and automated measurements (milk control, body weight).

BOCQUIER F., GUILLOUET P., BARILLET F., 1995. Alimentation hivernale des brebis laitières: intérêt de la mise en lots. INRA Prod. Anim., 8 (1), 19-28. 